\title{
Color Transparency and Saturation in $\mathrm{QCD}^{1}$
}

\author{
Dieter Schildknecht ${ }^{2}$ \\ Fakultät für Physik, Universität Bielefeld, \\ Universitätsstraße 25, 33615 Bielefeld, Germany \\ and \\ Max-Planck-Institute for Physics, Föhringer Ring 6, \\ 80805 Munich, Germany
}

\begin{abstract}
We review the theoretical interpretation of deep-inelastic electron-proton scattering at low values of the Bjorken variable $x \simeq Q^{2} / W^{2} \lesssim 0.1$. The process proceeds via the interaction of quark-antiquark $(q \bar{q})$ color-dipole fluctuations of the (virtual) photon with the proton. In terms of the forward Compton scattering amplitude, two reaction channels contribute to the interaction of the $q \bar{q}$ color dipole with the gluon field in the proton. Dependent on the kinematics, there is either color transparency, corresponding to a cancellation of the amplitudes for the two reaction channels, or saturation, occuring when the process is dominated by a single interaction channel. The connection between the color-dipole picture and the pQCD improved parton model is elaborated upon.
\end{abstract}

\section{Introduction}

The concepts of color transparency and saturation in QCD became relevant in connection with the interpretation of the deep inelastic scattering (DIS) experiments carried out at the $e^{ \pm}$-proton collider in Hamburg in the years from 1992 to 2007. The available centerof-mass energy $W$ allowed to explore DIS in a previously non-accessible range of very low values of the Bjorken variable $x \equiv x_{b j} \simeq Q^{2} / W^{2}$,

$$
5 \cdot 10^{-4} \leq x \leq 10^{-1}
$$

combined with a nevertheless appreciable range of the four-momentum squared of the virtual photon, approximately given by

$$
0 \leq Q^{2} \leq 100 G e V^{2}
$$

One of the outstanding findings from the HERA experiments is the low-x scaling behavior of the photoabsorption cross section, $\sigma_{\gamma^{*} p}\left(W^{2}, Q^{2}\right)$. When plotting, compare Fig. 1, the experimental data for $\sigma_{\gamma^{*} p}\left(W^{2}, Q^{2}\right)$ as a function of the low-x scaling variable

$$
\eta\left(W^{2}, Q^{2}\right)=\frac{Q^{2}+m_{0}^{2}}{\Lambda_{\text {sat }}^{2}\left(W^{2}\right)},
$$

\footnotetext{
${ }^{1}$ Presented at the International School of Subnuclear Physics, 50th Course, Erice, Sicily, 23 June - 2 July 2012

${ }^{2}$ Email: schild@physik.uni-bielefeld.de
} 
one finds a single curve [1, 2],

$$
\sigma_{\gamma^{*} p}\left(W^{2}, Q^{2}\right)=\sigma_{\gamma^{*} p}\left(\eta\left(W^{2}, Q^{2}\right)\right) \sim \sigma^{(\infty)} \begin{cases}\frac{1}{\eta\left(W^{2}, Q^{2}\right)}, & \text { for }\left(\eta\left(W^{2}, Q^{2}\right) \gg 1\right), \\ \ln \frac{1}{\eta\left(W^{2}, Q^{2}\right)}, & \text { for }\left(\eta\left(W^{2}, Q^{2}\right) \ll 1\right) .\end{cases}
$$

In $(1.3)$, the "saturation scale" $\Lambda_{\text {sat }}^{2}\left(W^{2}\right)$ rises with a small constant power, $C_{2}$, of the energy,

$$
\Lambda_{\text {sat }}^{2}\left(W^{2}\right) \sim\left(W^{2}\right)^{C_{2}}
$$

The value of $m_{0}$ is somewhat smaller than the mass of the $\rho^{0}$ meson. The quantity $\sigma^{(\infty)}$

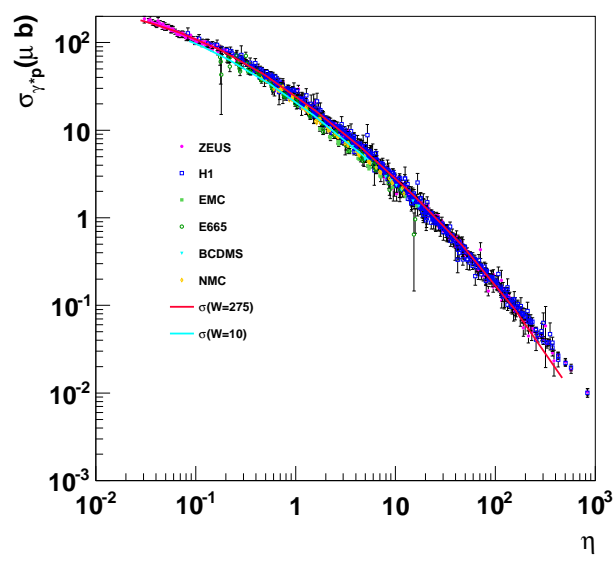

Figure 1: Scaling [1, 2] of $\sigma_{\gamma^{*} p}\left(W^{2}, Q^{2}\right)=\sigma_{\gamma^{*} p}\left(\eta\left(W^{2}, Q^{2}\right)\right)$.

denotes a cross section that in good approximation is independent of the photon energy. The log-log plot of the experimental data in Fig. 1 immediately allows one to infer the $1 / \eta\left(W^{2}, Q^{2}\right)$ dependence for $\eta\left(W^{2}, Q^{2}\right) \gg 1$ in 1.4$)$, as well as the $\ln \left(1 / \eta\left(W^{2}, Q^{2}\right)\right.$ dependence on $\eta\left(W^{2}, Q^{2}\right)$ for $\eta\left(W^{2}, Q^{2}\right) \ll 1$.

The logarithmic behavior in the limit of $\eta\left(W^{2}, Q^{2}\right) \rightarrow 0$, or $W^{2} \rightarrow \infty$ at $Q^{2}$ fixed, leads to the conclusion that the ratio of the virtual to the real photoabsorption cross section tends towards unity in this "saturation" limit [1, 2],

$$
\lim _{\substack{W^{2} \rightarrow \infty \\ Q^{2} \text { fixed }}} \frac{\sigma_{\gamma^{*} p}\left(\eta\left(W^{2}, Q^{2}\right)\right)}{\sigma_{\gamma^{*} p}\left(\eta\left(W^{2}, Q^{2}=0\right)\right)}=\lim _{\substack{W^{2} \rightarrow \infty \\ Q^{2} \text { fixed }}} \frac{\ln \left(\frac{\Lambda_{s a t}^{2}\left(W^{2}\right)}{m_{0}^{2}} \frac{m_{0}^{2}}{\left(Q^{2}+m_{0}^{2}\right)}\right)}{\ln \frac{\Lambda_{s a t}^{2}\left(W^{2}\right)}{m_{0}^{2}}}=1+\lim _{\substack{W^{2} \rightarrow \infty \\ Q^{2} \text { fixed }}} \frac{\ln \frac{m_{0}^{2}}{Q^{2}+m_{0}^{2}}}{\ln \frac{\Lambda_{s a t}^{2}\left(W^{2}\right)}{m_{0}^{2}}}=1 .
$$

The dependence of the photoabsorption cross section in (1.4) and (1.6) corresponds to a simple subdivision of the $\left(Q^{2}, W^{2}\right)$ plane. It is shown in Fig. 2. The region of $\eta\left(W^{2}, Q^{2}\right) \ll 1$, corresponds to "saturation" in the sense of an approach to the $Q^{2}=0$ photoabsorption cross section, while the region of $\eta\left(W^{2}, Q^{2}\right) \gg 1$ corresponds to "color transparency". The choice of this latter terminology will be justified in Section 3 below.

It is the aim of the present lecture to show that the scaling behavior with the functional form in (1.4) arises as a consequence of the color-gauge-invariant interaction of $q \bar{q}$ colordipole fluctuations of the photon with the proton (color-dipole picture, (CDP)). No specific parameter-dependent assumption on the dipole-proton interaction is needed to arrive at this conclusion. 


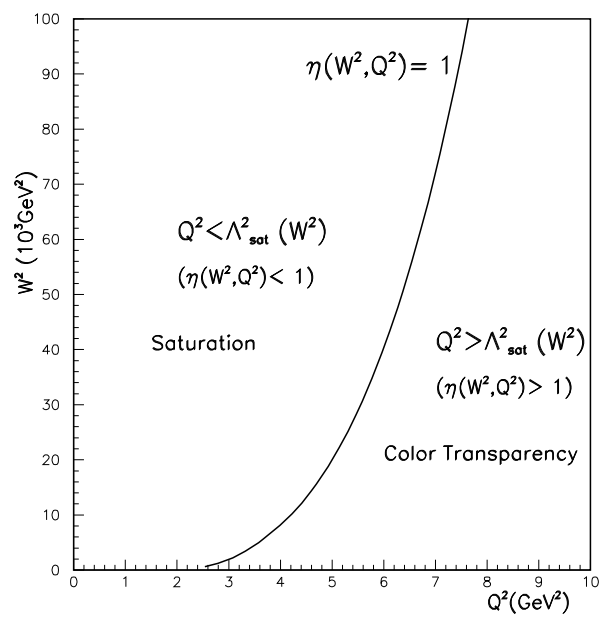

Figure 2: The $\left(Q^{2}, W^{2}\right)$ plane corresponding to the scaling behavior of $\sigma_{\gamma^{*} p}\left(W^{2}, Q^{2}\right)=$ $\sigma_{\gamma^{*} p}\left(\eta\left(W^{2}, Q^{2}\right)\right)$ shown in Fig. 1. The line $\eta\left(W^{2}, Q^{2}\right)=1$ subdivides the $\left(Q^{2}, W^{2}\right)$ plane into the saturation region of $\eta\left(W^{2}, Q^{2}\right)<1$ and the color transparency region of $\eta\left(W^{2}, Q^{2}\right)>1$.

Again, without parameter adjustment, the CDP leads to a unique prediction [3, 4] for the ratio $R\left(W^{2}, Q^{2}\right)$ of the longitudinal to the transverse photoabsorption cross section at sufficiently large $Q^{2}>\Lambda_{\text {sat }}^{2}\left(W^{2}\right)$. The definite value of $R\left(W^{2}, Q^{2}\right)$ arises as a consequence of the different transition probabilities for longitudinally polarized and transversely polarized photons into $q \bar{q}$ states, combined with the different transverse interaction size of $q \bar{q}$ states originating from longitudinally versus transversely polarized photons.

Consistency of the CDP with the pQCD-improved parton model allows one to deduce [2, 5, 6, 4] the numerical value of the exponent $C_{2}$ of the saturation scale $\Lambda_{\text {sat }}^{2}\left(W^{2}\right)$.

It is sometimes argued that the DIS data provide empirical evidence for parton recombination requiring non-linear parton evolution mechanisms (compare e.g. ref. [7] and the list of references therein). We do not see such an evidence.

\section{Photon hadron interactions: Late 1960's and early 1970's}

The general features of our present understanding of photon-hadron interactions at low values of $x \simeq Q^{2} / W^{2} \ll 0.1$ date back to the pre-QCD era of the late 1960's and early 1970's. So let me briefly return to that time.

Photon-hadron interactions in the late 1960's and early 1970's were dominated by the vector-meson-dominance (VMD) picture [8]-[12]: the photon virtually dissociates, or fluctuates in modern jargon, into the low-lying vector mesons, $\rho^{0}, \omega, \phi$, that subsequently interact with e.g. the proton. Compare Fig. 3, showing the specific case of (diffractive) vector meson production in photon-proton interactions. Inserting the $\gamma\left(\rho^{0}, \omega, \phi\right)$ couplings that had become available from $e^{+} e^{-}$-annihilation experiments in the late 1960's, and relating the vector-meson scattering amplitudes to the ones from pion-nucleon scattering 


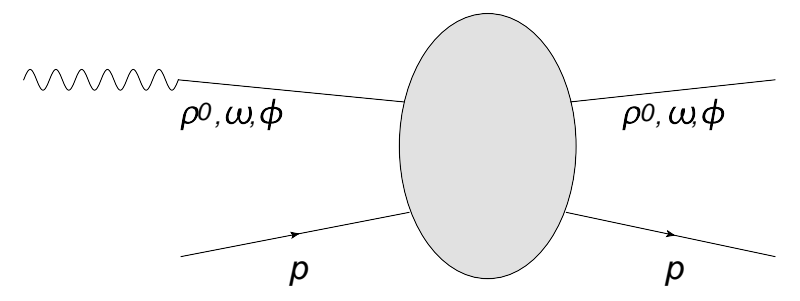

Figure 3: Vector-meson production in photon-proton interactions.

by invoking the additive quark model, vector meson production was quantitatively predicted as a function of energy and momentum transfer. The total photoproduction cross section then followed from the optical theorem by attaching a photon to the final vector mesons in Fig. 3. The experimental findings were theoretically summarized in terms of a "hadronlike behavior" [13, 14] of the photon.

Based on vector-meson dominance, hadronlike behavior of the cross section of photoproduction on heavy nuclei was predicted [15] as a consequence of the destructive interference of the one- and two-channel interactions depicted in Fig. 4. Experiments at DESY and SLAC confirmed the theoretical prediction of a hadronlike dependence on the mass number of the nucleus ("shadowing").

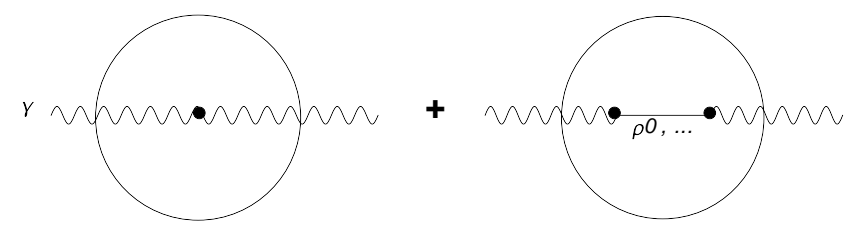

Figure 4: The forward-Compton-scattering amplitude of the interaction of a photon with a heavy nucleus.

The picture of photon-hadron interactions changed dramatically, when, starting in 1969, the first experimental results on DIS at large values of $Q^{2}$ from the SLAC-MIT collaboration became available [16]. The experimental results provided evidence for the scaling behavior of the proton structure function that had been conjectured by Bjorken [17, and they led Feynman to propose the parton-model [18].

An alternative point of view concerning the results from the SLAC-MIT experiment was advocated by Sakurai and myself in 1972 [19]. Since this point of view is of much relevance as a predecessor of our present understanding of DIS at low $x \equiv x_{b j}$ in QCD, I briefly remind you of our 1972 procedure.

We conjectured that the slow decrease of the photoabsorption cross section with increasing $Q^{2}$ observed by the SLAC-MIT collaboration at large $w^{\prime} \sim 1 / x$ was due to the, at that time hypothetica ${ }^{3}$, coupling of the photon to a high-mass continuum predicted to

\footnotetext{
${ }^{3}$ First experimental data on $e^{+} e^{-}$annihilation beyond the $\rho^{0}, \omega, \phi$ region had become available [20] from the Frascati $e^{+} e^{-}$storage ring (Adone) in 1971, providing evidence for production of multi-hadron final states at $1.3 \mathrm{GeV}$ to $1.4 \mathrm{GeV} e^{+} e^{-}$center-of-mass energy.
} 
be observed in future $e^{+} e^{-}$annihilation experiments beyond the $\rho^{0}, \omega, \phi$ region. Compare Fig. 5 and the comparison of our generalized vector dominance (GVD) predictions [19]

$$
r^{*} \aleph_{\rho^{0, \omega, \phi}}+r^{*} \mathrm{~W}_{\substack{\text { massive } \\ \text { continuum }}}
$$

Figure 5: The coupling of the photon to a high-mass continuum.

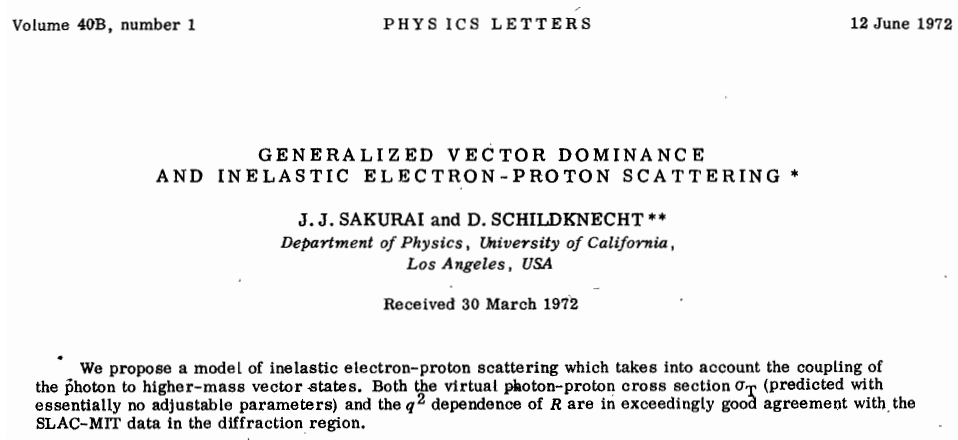

with the experimental data in Fig. 6. The GVD approach was further developed in ref. [21], compare also [22] and the review in ref. [12].
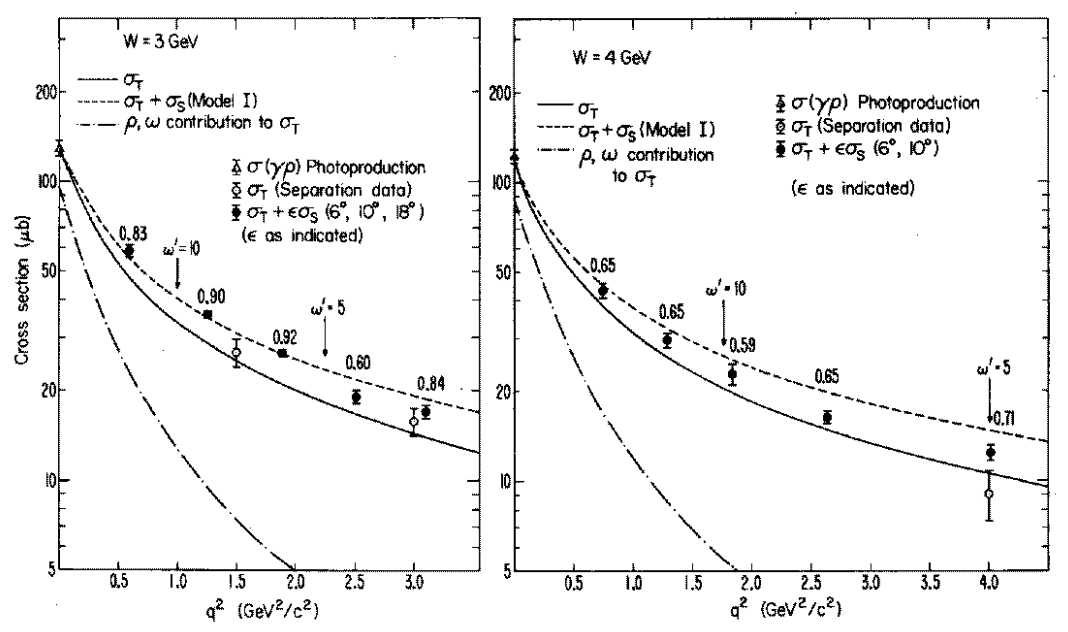

Figure 6: The SLAC-MIT experimental data compared with GVD predictions [19].

In the early 1970's, it was frequently argued that our GVD interpretation of the experimental results was incorrect, since experiments did not reveal the hadronlike shadowing 
in the scattering of virtual photons from complex nuclei implied by GVD. After many years of confusion, this point was clarified by the 1989 experimental results of the EMCNMC collaboration [23], compare Fig. 7. The results indeed showed the shadowing effect expected for virtual photons due to high-mass fluctuations [24] of the virtual photon that contribute to the two-step interaction in Fig. 4.

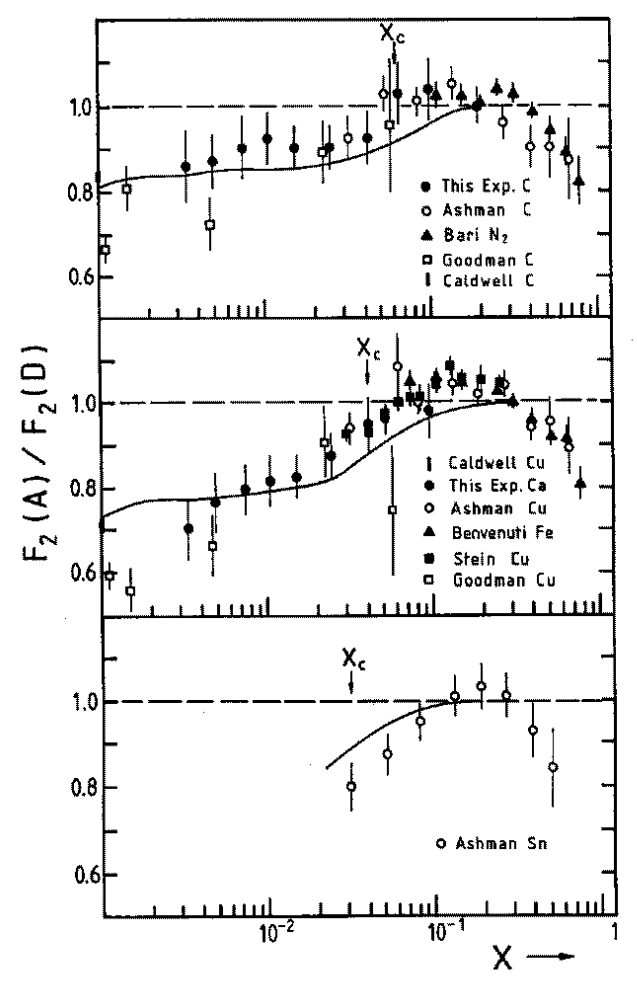

Figure 7: Shadowing in the scattering of virtual photons from complex nuclei compared with GVD predictions [24]

Since shadowing for virtual photons requires high-mass diffractive production, contrary to what is often stated, it came without surprise that one of the first results from HERA was the observation of "rap-gap" events [25] due to diffractive production of high-mass hadron states.

The 1972 approach of GVD [19] was based on postulating a mass dispersion relation as immediate generalization of $\left(\rho^{0}, \omega, \phi\right)$-dominance,

$$
\sigma_{\gamma_{T}^{*} p}\left(W^{2}, Q^{2}\right)=\int d m^{2} \int d m^{\prime 2} \frac{\rho\left(W^{2}, m^{2}, m^{\prime 2}\right) m^{2} m^{\prime 2}}{\left(Q^{2}+m^{2}\right)\left(Q^{2}+m^{\prime 2}\right)} .
$$

The momentum space dispersion-theoretic ansatz may be equivalently interpreted in terms of a three-momentum conserving energy-conservation-violating transition to an on-shell hadronic state of finite, but sufficiently long lifetime given by [26, 24]

$$
\tau=\frac{1}{\Delta E}=\frac{2 M \nu}{Q^{2}+M_{q \bar{q}}^{2}} \frac{1}{M_{p}}=\frac{1}{x+\frac{M_{q \bar{q}}^{2}}{W^{2}}} \frac{1}{M_{p}} \gg \frac{1}{M_{p}},
$$


followed by the interaction of the hadronic fluctuation with the nucleon. In $(2.2), \nu$ denotes the proton-rest-frame energy of the photon and $M_{q \bar{q}}$ and $M_{p}$ denote the fluctuation mass and the proton mass, respectively.

\section{The modern picture of DIS at low x: the color dipole picture}

As in GVD, in the modern approach, the photoabsorption reaction proceeds in two steps, i) the $\gamma^{*} \rightarrow q \bar{q}$ transition and ii) the $(q \bar{q})$-proton interaction, whereby taking into account

i) the internal structure of the $q \bar{q}$ system via the variable $0 \leq z \leq 1$ that specifies the longitudinal momentum distribution between the quark and the antiquark the photon fluctuates into, compare Fig. 8, and

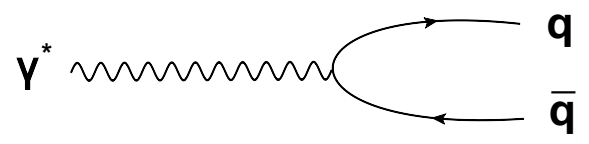

Figure 8: The $\gamma^{*} q \bar{q}$ transition

ii) the $q \bar{q}$ interaction with the gluon field in the nucleon [27] as a gauge-invariant colordipole interaction, compare the (virtual) forward Compton scattering amplitude in Fig. 9.

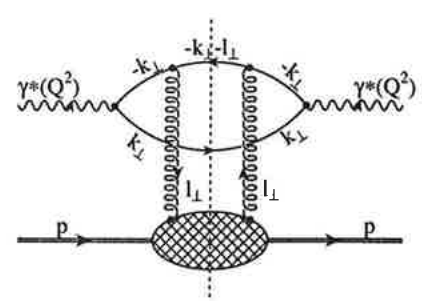

(a)

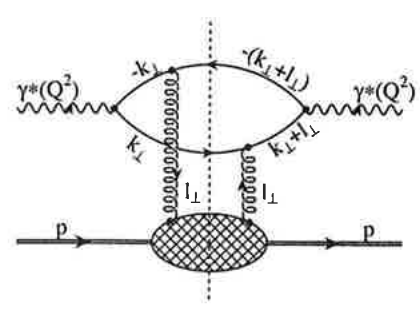

(b)

Figure 9: Two of the four diagrams for the $q \bar{q}$ dipole interaction with the gluon field in the nucleon. The diagrams (a) and (b) correspond to channel 1 and channel 2 respectively.

After Fourier transform to transverse position space, the photoabsorption cross section at low $x \simeq Q^{2} / W^{2}<0.1$ takes the form [28, 29]

$$
\sigma_{\gamma_{L, T}^{*}}\left(W^{2}, Q^{2}\right)=\int d z \int d^{2} \vec{r}_{\perp}\left|\psi_{L, T}\left(\vec{r}_{\perp}, z(1-z), Q^{2}\right)\right|^{2} \cdot \sigma_{(q \bar{q}) p}\left(\vec{r}_{\perp}, z(1-z), W^{2}\right) .
$$

The quantity $\left|\psi_{L, T}\left(\vec{r}_{\perp}, z(1-z), Q^{2}\right)\right|^{2}$ may be interpreted as the probability for a longitudinally or a transversely polarized photon, $\gamma_{L, T}^{*}$, of virtuality $Q^{2}$ to undergo a transition to a $q \bar{q}$ state, $\gamma_{L, T}^{*} \rightarrow q \bar{q}$, being characterized by the transverse size $\vec{r}_{\perp}$ and by the distribution of the longitudinal momenta of quark and antiquark determined by $z(1-z)$. In the rest frame of the $q \bar{q}$ fluctuation of mass $M_{q \bar{q}}$, the quantity $z(1-z)$ determines [29] the 
direction of the quark (antiquark) with respect to the photon direction. The interaction cross section of the $q \bar{q}$ dipole state in $(3.1)$ is denoted by $\sigma_{(q \bar{q}) p}\left(\vec{r}_{\perp}, z(1-z), W^{2}\right)$. It depends on the energy, $W,[29,1,30,31]$ of the $(q \bar{q}) p$ interaction, since the photon fluctuates into an on-shell $q \bar{q}$ state of mass $M_{q \bar{q}}$ that subsequently interacts with the nucleon. For generality, a dependence on $z(1-z)$ is allowed for in the dipole-proton cross section.

The gauge-invariant two-gluon interaction ii) of the $q \bar{q}$ dipole enters the photoabsorption cross section in (3.1) via [28, 29]

$$
\sigma_{(q \bar{q}) p}\left(\vec{r}_{\perp}, z(1-z), W^{2}\right)=\int d^{2} \vec{l}_{\perp} \tilde{\sigma}\left(\vec{l}_{\perp}^{2}, z(1-z), W^{2}\right)\left(1-e^{-i \vec{l}_{\perp} \cdot \vec{r}_{\perp}}\right)
$$

In $3.2, \vec{l}_{\perp}$ stands for the transverse momentum of the absorbed gluon, and the first and the second term in the parenthesis on the right-hand side in (3.2), respectively, corresponds to the first and the second diagram in Fig. 9.

For the subsequent discussions, it will be useful to equivalently rewrite [32, 4] the photoabsorption cross section (3.1), in terms of dipole states, $(q \bar{q})_{L, T}^{J=1}$, that describe longitudinally and transversely polarized $q \bar{q}$ states of fixed spin $J=1$ and polarization index $L$ and $T$. In terms of the corresponding dipole cross section, $\sigma_{(q \bar{q})_{L, T}^{J=1} p}\left(\vec{r}_{\perp}^{\prime}, W^{2}\right)$, where $\vec{r}_{\perp}^{\prime}=\vec{r}_{\perp} z(1-z)$, the photoabsorption cross section (3.1) becomes [32, 4 ]

$$
\sigma_{\gamma_{L, T}^{*} p}\left(W^{2}, Q^{2}\right)=\frac{\alpha}{\pi} \sum_{q} Q_{q}^{2} Q^{2} \int d r_{\perp}^{\prime 2} K_{0,1}^{2}\left(r_{\perp}^{\prime} Q\right) \sigma_{(q \bar{q}) L, T}^{J=1} p\left(r_{\perp}^{\prime 2}, W^{2}\right)
$$

In the transition from (3.1) to (3.3), assuming massless quarks, we inserted the explicit expressions for $\left|\psi_{L, T}\left(\vec{r}_{\perp}, z(1-z), Q^{2}\right)\right|^{2}$ in terms of the modified Bessel functions $K_{0}\left(r_{\perp}^{\prime} Q\right)$ and $K_{1}\left(r_{\perp}^{\prime} Q\right)$, and $Q$ stands for $Q \equiv \sqrt{Q^{2}}$. The sum over the squared charges of the actively contributing quarks is given by $\sum_{q} Q_{q}^{2}$, and the cross section $\sigma_{(q \bar{q})_{L, T}^{J=1}}\left(r_{\perp}^{\prime}, W^{2}\right)$ is related to the dipole cross section in (3.1) by an appropriate projection.

In terms of the $J=1$ projection, $\sigma_{(q \bar{q})_{L, T}^{J=1} p}\left(r_{\perp}^{\prime}, W^{2}\right)$, of the dipole cross section in (3.1), with $\vec{l}_{\perp}^{\prime 2}=\vec{l}_{\perp}^{2} / z(1-z)$, the two-gluon-coupling structure of the dipole cross section in (3.2) becomes [4]

$\sigma_{(q \bar{q})_{L, T}^{J=1} p}\left(r_{\perp}^{\prime}, W^{2}\right)=\pi \int d \vec{l}_{\perp}^{\prime 2} \bar{\sigma}_{(q \bar{q})_{L, T}^{J=1} p}\left(\vec{l}_{\perp}^{\prime 2}, W^{2}\right) \cdot\left(1-\frac{\int d \vec{l}_{\perp}^{\prime 2} \bar{\sigma}_{(q \bar{q})_{L, T}^{J=1} p}\left(\vec{l}_{\perp}^{\prime 2}, W^{2}\right) J_{0}\left(l_{\perp}^{\prime} r_{\perp}^{\prime}\right)}{\int d \vec{l}_{\perp}^{\prime 2} \bar{\sigma}_{(q \bar{q})_{L, T}^{J=1} p}\left(\vec{l}_{\perp}^{\prime 2}, W^{2}\right)}\right)$,

where $J_{0}\left(l_{\perp}^{\prime} r_{\perp}^{\prime}\right)$ denotes the Bessel function with index 0 .

Two distinct $W$-dependent limits of the dipole cross section (3.4) will be relevant [4] and important for the ensuing discussions. We assume that the integrals in (3.4) exist and are determined by a $W$-dependent restricted range of $\vec{l}_{\perp}^{\prime 2}<\vec{l}_{\text {Max }}^{\prime 2}\left(W^{2}\right)$, in which $\bar{\sigma}_{(q \vec{q})_{L, T}^{J=1} p}\left(\vec{l}_{\perp}^{\prime 2}, W^{2}\right)$ is appreciably different from zero, $\vec{l}_{\perp \text { Max }}^{\prime 2}\left(W^{2}\right)$ increasing with increasing $W^{2}$. The resulting cross section for a dipole of fixed transverse size, $r_{\perp}^{\prime}$, strongly depends on the variation of the phase $l_{\perp}^{\prime} r_{\perp}^{\prime}$ :

A) For

$$
0<l_{\perp}^{\prime} r_{\perp}^{\prime}<l_{\perp \operatorname{Max}}^{\prime}\left(W^{2}\right) r_{\perp}^{\prime} \ll 1
$$


upon employing the expansion

$$
J_{0}\left(l_{\perp}^{\prime} r_{\perp}^{\prime}\right) \cong 1-\frac{1}{4}\left(l_{\perp}^{\prime} r_{\perp}^{\prime}\right)^{2}+\frac{1}{4^{3}}\left(l_{\perp}^{\prime} r_{\perp}^{\prime}\right)^{4}+\cdots
$$

we find a strong cancellation between the two additive contributions to the $J=1$ dipole cross section (3.4) which correspond to the first and the second diagram in Fig. 9. The dipole cross section (3.4) becomes proportional to $r_{\perp}^{\prime 2}$ ("color transparency" limit [28])

$$
\sigma_{(q \bar{q}) J_{L, T}^{J=1} p}\left(r_{\perp}^{\prime 2}, W^{2}\right)=\frac{1}{4} r_{\perp}^{\prime 2} \sigma_{L}^{(\infty)}\left(W^{2}\right) \Lambda_{s a t}^{2}\left(W^{2}\right)\left\{\begin{array}{c}
1, \\
\rho_{W},
\end{array} \quad\left(r_{\perp}^{\prime 2} \ll \frac{1}{l_{\perp M a x}^{\prime 2}\left(W^{2}\right)}\right)\right.
$$

where by definition the $W^{2}$-dependent scale $\Lambda_{\text {sat }}^{2}\left(W^{2}\right)$ reads

$$
\Lambda_{\text {sat }}^{2}\left(W^{2}\right) \equiv \frac{1}{\sigma_{L}^{(\infty)}\left(W^{2}\right)} \pi \int d \vec{l}_{\perp}^{\prime 2} \vec{l}_{\perp}^{\prime 2} \bar{\sigma}_{(q \bar{q})_{L}^{J=1} p}\left(\vec{l}_{\perp}^{\prime 2}, W^{2}\right)
$$

and $\sigma_{L}^{(\infty)}\left(W^{2}\right)$ is explicitly defined by 3.11 below. For vanishing size, $\vec{r}_{\perp}^{\prime 2}$, the $q \bar{q}$ color dipole (obviously) has a vanishing cross section. The expression for the factor $\rho_{W}$ in (3.7) is explicitly obtained by comparison of (3.7) with (3.4). According to (3.7), and anticipating $\rho_{W}>1$, transversely polarized $(q \bar{q})^{J=1}$ states interact with enhanced transverse size [3, 4]

$$
\vec{r}_{\perp}^{\prime 2} \rightarrow \rho_{W} \vec{r}_{\perp}^{\prime 2}
$$

relative to longitudinal ones. The ratio $\rho_{W}$ will be shown to be a $W$-independent constant of definite magnitude, $\rho_{W}=\rho=4 / 3$.

B) For the case of

$$
l_{\perp \operatorname{Max}}^{\prime}\left(W^{2}\right) r_{\perp}^{\prime} \gg 1,
$$

alternative to 3.5$)$, for any fixed value of $r_{\perp}^{\prime}$, rapid oscillations of the Bessel function in (3.4) lead to a vanishingly small contribution of the second term in (3.4) thus implying ("saturation" limit)

$$
\lim _{\substack{r_{f i x e d} \\ W^{2} \rightarrow \infty}} \sigma_{(q \bar{q})_{L, T}^{J=1} p}\left(r_{\perp}^{\prime 2}, W^{2}\right)=\pi \int d \vec{l}_{\perp}^{\prime 2} \bar{\sigma}_{(q \bar{q})_{L, T}^{J=1} p}\left(\vec{l}_{\perp}^{\prime 2}, W^{2}\right),\left(r_{\perp}^{\prime 2} \gg \frac{1}{l_{\perp M a x}^{\prime 2}\left(W^{2}\right)}\right) .
$$

The high-energy limit in (3.11) of sufficiently large $W$ at fixed dipole size $r_{\perp}^{\prime}$, according to 3.4 , coincides with the limit of sufficiently large $r_{\perp}^{\prime}$ at fixed $W$ i.e.

$$
\lim _{\substack{r_{\text {fixed }}^{\prime 2} \\ W^{2} \rightarrow \infty}} \sigma_{(q \bar{q})_{L, T}^{J=1} p}\left(r_{\perp}^{\prime 2}, W^{2}\right)=\lim _{\substack{r^{\prime 2} \rightarrow \infty \\ W^{2}=\text { const }}} \sigma_{(q \bar{q})_{L, T}^{J=1}}\left(r_{\perp}^{\prime 2}, W^{2}\right)=\sigma_{L, T}^{(\infty)}\left(W^{2}\right) .
$$

At fixed dipole size, at sufficiently large energy, we arrive at the large-dipole-size limit of the (at most weakly $W$-dependent) hadronic cross section $\sigma_{L, T}^{(\infty)}\left(W^{2}\right) \simeq \sigma^{(\infty)}$. 
The photoabsorption cross section in (3.3), due to the strong decrease of the modified Bessel functions $K_{0,1}\left(r_{\perp}^{\prime} Q\right)$ with increasing argument $r_{\perp}^{\prime} Q$, is strongly dominated and actually determined at any fixed value of $Q^{2}$ by values of $r_{\perp}^{\prime 2}$ such that $r_{\perp}^{\prime 2} Q^{2}<1$. Whether color transparency of the dipole cross section according to (3.5) and (3.7) or, alternatively, saturation according to 3.10 and $\sqrt{3.11}$ is relevant for $\sigma_{\gamma_{L, T}^{*}}\left(W^{2}, Q^{2}\right)$ in (3.3) depends on whether $Q^{2} \gg \Lambda_{\text {sat }}^{2}\left(W^{2}\right)$ or $Q^{2} \ll \Lambda_{\text {sat }}^{2}\left(W^{2}\right)$ is realized at a specific value of $W$. Upon substitution of (3.7) and, alternatively, of (3.11) into (3.3), for $\sigma_{\gamma^{*} p}\left(W^{2}, Q^{2}\right)=$ $\sigma_{\gamma_{L}^{*} p}\left(W^{2}, Q^{2}\right)+\sigma_{\gamma_{T}^{*} p}\left(W^{2}, Q^{2}\right)$ one finds [1, 4]

$\sigma_{\gamma^{*} p}\left(W^{2}, Q^{2}\right)=\sigma_{\gamma^{*} p}\left(\eta\left(W^{2}, Q^{2}\right)\right)=\frac{\alpha}{\pi} \sum_{q} Q_{q}^{2} \cdot \begin{cases}\frac{1}{6}(1+2 \rho) \sigma^{(\infty)} \frac{1}{\eta\left(W^{2}, Q^{2}\right)}, & \left(\eta\left(W^{2}, Q^{2}\right) \gg 1\right), \\ \sigma^{(\infty)} \ln \frac{1}{\eta\left(W^{2}, Q^{2}\right)}, & \left(\eta\left(W^{2}, Q^{2}\right) \ll 1\right),\end{cases}$

in agreement with the experimental result (1.4) shown in Fig. 1. In (3.13), we have introduced the low-x scaling variable [1]

$$
\eta\left(W^{2}, Q^{2}\right)=\frac{Q^{2}+m_{0}^{2}}{\Lambda_{\text {sat }}^{2}\left(W^{2}\right)}
$$

and anticipated $\rho_{W}=$ const. $=\rho$. In (3.14), via quark-hadron duality [19, 33], we introduced the lower bound $m_{0}^{2} \lesssim m_{\rho}^{2}$ on the masses of the $q \bar{q}$ fluctuations $M_{q \bar{q}}^{2} \geq m_{0}^{2}$, only relevant in the limit of $Q^{2} \rightarrow 0$.

From the above derivation, leading to (3.13), it has become clear that DIS at low $\mathrm{x}$ in terms of the Compton-forward-scattering amplitude proceeds via two different reaction channels. They correspond to the first and the second diagram in Fig. 9. For sufficiently large $Q^{2} \gg \Lambda_{\text {sat }}^{2}\left(W^{2}\right)$ both channels are open, resulting in strong cancellation between them. With decreasing $Q^{2}$ at fixed $W^{2}$, or with increasing $W^{2}$ at fixed $Q^{2}$, for $Q^{2} \ll$ $\Lambda_{\text {sat }}^{2}\left(W^{2}\right)$, the second channel becomes closed, no cancellation any more. Only the first channel remains open, implying that the proportionality of the photoabsorption cross section to $\Lambda_{\text {sat }}^{2}\left(W^{2}\right)$ turns into the (soft) energy dependence proportional to $\ln \Lambda_{\text {sat }}^{2}\left(W^{2}\right)$, compare 3.13 .

The longitudinal-to-transverse ratio of the photoabsorption cross sections $\sigma_{\gamma_{L}^{*} p}\left(W^{2}, Q^{2}\right)$ and $\sigma_{\gamma_{T}^{*} p}\left(W^{2}, Q^{2}\right)$ at large $Q^{2} \gg \Lambda_{\text {sat }}^{2}\left(W^{2}\right)$ according to 3.13 is given by

$$
R\left(W^{2}, Q^{2}\right)_{Q^{2} \gg \Lambda_{\text {sat }}^{2}\left(W^{2}\right)}=\left.\frac{\sigma_{\gamma_{L}^{*}}\left(W^{2}, Q^{2}\right)}{\sigma_{\gamma_{T}^{*}}\left(W^{2}, Q^{2}\right)}\right|_{Q^{2} \gg \Lambda_{s a t}^{2}\left(W^{2}\right)}=\frac{1}{2 \rho} .
$$

The factor 2 in 3.15 originates from the difference in the photon wave functions in (3.3). The interaction with enhanced transverse size of $(q \bar{q})_{T}^{J=1}$ states relative to $(q \bar{q})_{L}^{J=1}$ states, $\rho_{W}$ in (3.9), is a consequence of the ratio of the average transverse momenta of the quark (antiquark) in the $(q \bar{q})_{T}^{J=1}$ state relative to the quark (antiquark) in the $(q \bar{q})_{L}^{J=1}$ state. Upon applying the uncertainty principle, one obtains [3, 4]

$$
\rho_{W}=\rho=\frac{4}{3} .
$$

The longitudinal structure function, with 3.15 and $(3.16)$, at large $Q^{2}$ is related to the transverse one via

$$
F_{L}\left(x, Q^{2}\right)=\frac{1}{1+2 \rho} F_{2}\left(x, Q^{2}\right)=0.27 F_{2}\left(x, Q^{2}\right) .
$$


The result is consistent with the experimental data, compare Fig. 10. In Fig. 10, in addition to the experimental results on $F_{L}$ from the $\mathrm{H} 1$ and ZEUS collaborations, we also show the result on $\left(F_{L} / F_{2}\right) /\left.\left(F_{L} / F_{2}\right)\right|_{\text {bound }}$ from a very detailed analysis of the experimental data by the authors of ref. [34]. Since the value of $\left.\left(F_{L} / F_{2}\right)\right|_{\text {bound }}=0.27$ from ref. [34] (accidentally) numerically coincides with our prediction (3.17), the analysis of ref. 34] provides additional confirmation, within errors, of the prediction (3.17).
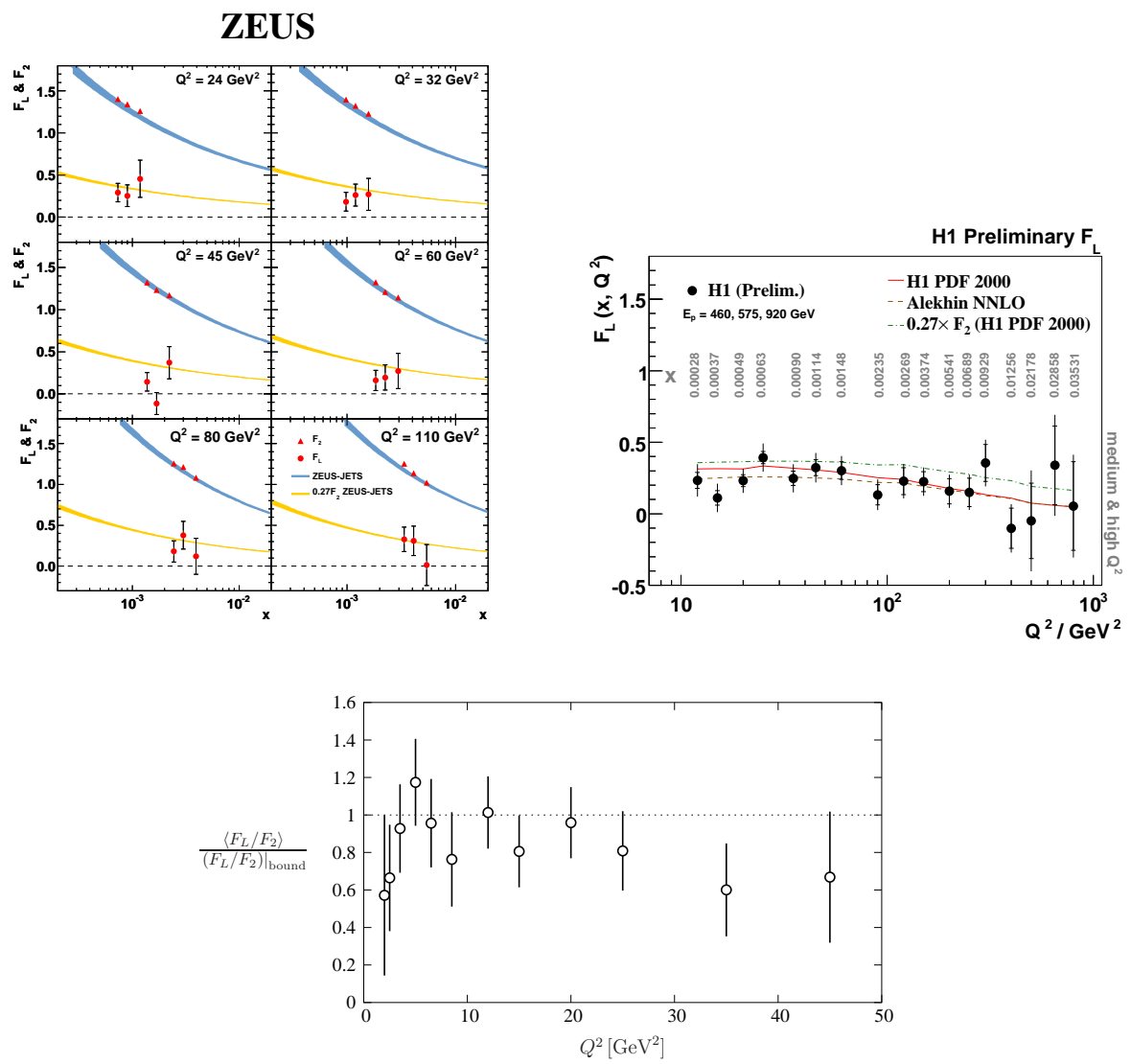

Figure 10: The prediction 3.17) compared with the experimental data for $F_{L}$ and $F_{2}$.

The $W$-dependence in (3.1) and (3.3) of the dipole cross section, combined with the $1 / Q^{2}$ dependence in 3.13 at large $Q^{2}$, implies that the structure function $F_{2}\left(x, Q^{2}\right) \simeq$ $\left(Q^{2} / 4 \pi^{2} \alpha\right)\left(\sigma_{\gamma_{L}^{*} p}\left(W^{2}, Q^{2}\right)+\sigma_{\gamma_{T}^{*} p}\left(W^{2}, Q^{2}\right)\right)$ becomes a function of the single variable $W^{2}$. The experimental data in Fig. 11, in the relevant range of $x \simeq Q^{2} / W^{2}<0.1$, approximately corresponding to $1 / W^{2} \leq 10^{-3}$, indeed show a single line [4] $]^{4}$ consistent with the representation of $\sigma_{\gamma^{*} p}\left(\eta\left(W^{2}, Q^{2}\right)\right)$ in Fig. 1. An eye-ball fit to the data in Fig. 11 yields

$$
F_{2}\left(W^{2}\right)=f_{2} \cdot\left(\frac{W^{2}}{1 \mathrm{GeV}^{2}}\right)^{C_{2}=0.29}
$$

with $f_{2}=0.063$. We shall come back to representation 3.18 of $F_{2}\left(W^{2}\right)$ below. For comparison, in Fig. 11, we also show $F_{2}\left(x, Q^{2}\right)$ as a function of the Bjorken variable $x$.

\footnotetext{
${ }^{4}$ The representation of the experimental data in Fig. 11 was kindly prepared by Prabhdeep Kaur.
} 

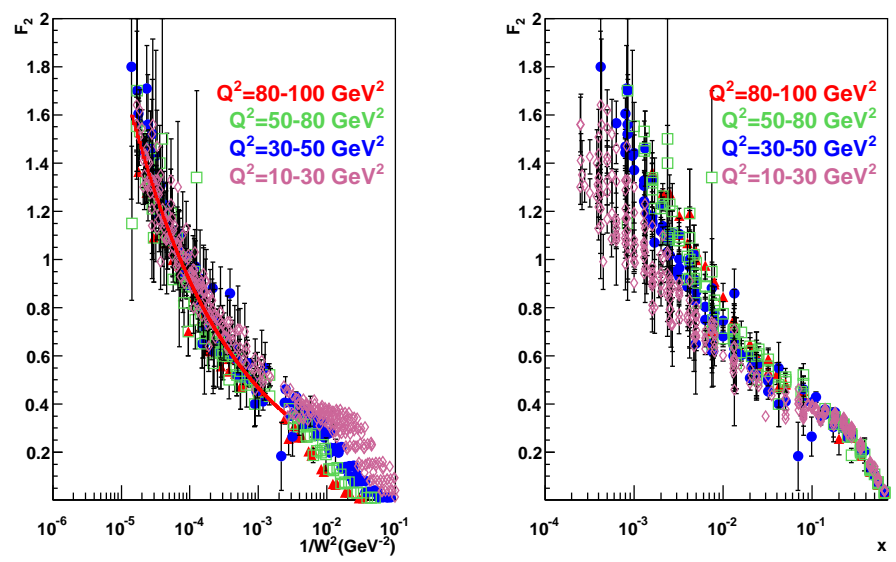

Figure 11: The structure function $F_{2}\left(x, Q^{2}\right)$ as a function of $1 / W^{2}$ and as a function of $x$

We turn to the limit of $\eta\left(W^{2}, Q^{2}\right) \ll 1$, or $W^{2} \rightarrow \infty$ at $Q^{2}$ fixed in 3.13, 1.4 and (1.6). The convergence to this limit is extremely slow. Compare Fig. 12, where this limit is shown in terms of the structure function $F_{2}\left(x, Q^{2}\right)$,

$$
\lim _{\substack{W^{2} \rightarrow \infty \\ Q^{2} \text { fixed }}} \frac{F_{2}\left(x \cong Q^{2} / W^{2}, Q^{2}\right)}{\sigma_{\gamma p}\left(W^{2}\right)}=\frac{Q^{2}}{4 \pi^{2} \alpha} .
$$

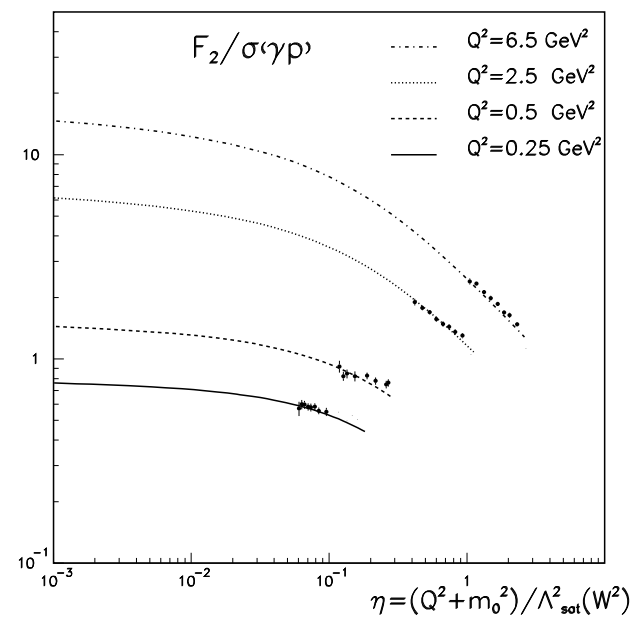

Figure 12: The approach to saturation

The theoretical curve in Fig. 12 is due to the concrete ansatz for the dipole cross section in Section 4 that interpolates between the regions of $\eta\left(W^{2}, Q^{2}\right) \gg 1$ and $\eta\left(W^{2}, Q^{2}\right) \ll 1$.

An approach to a $Q^{2}$-independent limit for $W^{2} \rightarrow \infty$ at fixed $Q^{2}$ was recently independently observed by Caldwell [35] based on a purely empirical fit to the experimental data given by

$$
\sigma_{\gamma^{*} p}\left(W^{2}, Q^{2}\right)=\sigma_{0}\left(Q^{2}\right)\left(\frac{1}{2 M_{p}} \frac{W^{2}}{Q^{2}}\right)^{\lambda_{e f f}\left(Q^{2}\right)} .
$$

The straight lines in Fig. 13 meet at a value of $W^{2}$ approximately given by $W^{2} \simeq 10^{9} Q^{2}$, consistent with the above conclusion from the CDP. 


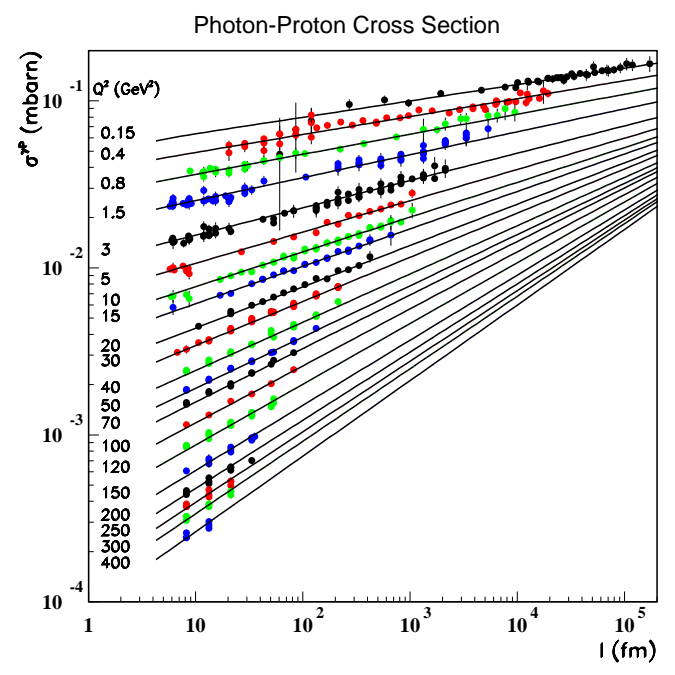

Figure 13: The Caldwell fit 3.20] 35]

The results from the above general analysis of the CDP lead to the simple structure of the $\left(Q^{2}, W^{2}\right)$ plane shown in Fig. 2 .

The $\left(Q^{2}, W^{2}\right)$ plane is subdivided into (only) two regions as a consequence of the two interaction channels corresponding to the diagrams in Fig. 9. For $\eta\left(W^{2}, Q^{2}\right)>1$, both interaction channels, channels 1 and 2, are open, implying color transparency of the $(q \bar{q}) p$ dipole interaction and strong destructive interference in the photoabsorption cross section for $\gamma^{*} p \rightarrow$ hadrons. For $\eta\left(W^{2}, Q^{2}\right) \ll 1$, channel 2 becomes closed. The lack of cancellation leads to a saturation of the cross section being determined by the dipole interaction solely via channel 1 . The virtual-photon-nucleon cross section approaches a $Q^{2}$-independent saturation limit that coincides with $Q^{2}=0$ photoproduction.

\section{The CDP, the gluon distribution function and evo- lution}

The CDP of DIS correspond $\mathrm{s}^{5}$ to the low $x$ approximation of the pQCD-improved parton model in which the interaction of the (virtual) photon occurs by interaction with the quark-antiquark sea in the proton via $\gamma^{*}$ gluon $\rightarrow q \bar{q}$ fusion.

The longitudinal structure function in this approximation, for a wide range of different gluon distributions, becomes proportional to the gluon density at a rescaled value $x / \xi_{L}$ 36

$$
F_{L}\left(\xi_{L} x, Q^{2}\right)=\frac{\alpha_{s}\left(Q^{2}\right)}{3 \pi} \sum_{q} Q_{q}^{2} G\left(x, Q^{2}\right),
$$

where $G\left(x, Q^{2}\right) \equiv x g\left(x, Q^{2}\right)$ and $g\left(x, Q^{2}\right)$ stands for the gluon-distribution function. The rescaling factor has the preferred value of $\xi_{L} \simeq 0.40$.

The structure function $F_{2}\left(x, Q^{2}\right)$ at low $x$ in this approximation is proportional to the sea-quark distribution, and again for a wide range of different gluon distributions, the

\footnotetext{
${ }^{5}$ With respect to this Section, compare also ref. 6]
} 
evolution of the structure function $F_{2}\left(x, Q^{2}\right)$ with $Q^{2}$ is determined by [37, 38]

$$
\frac{\partial F_{2}\left(\xi_{2} x, Q^{2}\right)}{\partial \ln Q^{2}}=\frac{\alpha_{s}\left(Q^{2}\right)}{3 \pi} \sum_{q} Q_{q}^{2} G\left(x, Q^{2}\right),
$$

where the preferred value of $\xi_{2}$ is given by $\xi_{2} \simeq 0.50$.

According to the CDP, compare (3.13) and (3.18), and supported by the experimental data in Fig. 11, the structure function $F_{2}\left(x, Q^{2}\right)$ for sufficiently large $Q^{2}$ becomes a function of the single variable $W^{2}$,

$$
F_{2}\left(x, Q^{2}\right)=F_{2}\left(W^{2}=\frac{Q^{2}}{x}\right) .
$$

Employing the proportionality of $F_{L}\left(x, Q^{2}\right)=\left(1 /\left(1+2 \rho_{W}\right)\right) F_{2}\left(x, Q^{2}\right)$ from (3.7) and (3.17), and combining (4.1) and (4.2), upon inserting (4.3) the evolution equation becomes

$$
\left(2 \rho_{W}+1\right) \frac{\partial}{\partial \ln W^{2}} F_{2}\left(\frac{\xi_{L}}{\xi_{2}} W^{2}\right)=F_{2}\left(W^{2}\right) .
$$

A potential dependence of $\rho_{W}$ on the energy $W$ is allowed in (4.4).

We specify $F_{2}\left(W^{2}\right)$ by adopting the power law [4]

$$
F_{2}\left(W^{2}\right) \sim\left(W^{2}\right)^{C_{2}}=\left(\frac{Q^{2}}{x}\right)^{C_{2}} .
$$

A power law in $(1 / x)^{\lambda}$ with $\lambda=$ const. occurs e.g. in the "hard Pomeron solution" [39] of DGLAP evolution as well as in the "hard Pomeron" part of Regge phenomenology with $(1 / x)^{\epsilon_{0}}$ and $\epsilon_{0} \simeq 0.43$ from a fit [40]. The CDP in (4.5) is more specific, however, since the $W$ dependence of $F_{2}\left(W^{2}\right)$ implies that the $x$ dependence and the $Q^{2}$ dependence are intimately related to each other.

Substitution of 4.5) into 4.4) implies the constraint [5, 4]

$$
\left(2 \rho_{W}+1\right) C_{2}\left(\frac{\xi_{L}}{\xi_{2}}\right)^{C_{2}}=1 .
$$

If, and only if $\rho_{W}=\rho=$ const., also the exponent is constant, $C_{2}=$ const. With the CDP result of $\rho=4 / 3$ from $(3.16)$, we obtain the unique value of

$$
C_{2}=\frac{1}{2 \rho+1}\left(\frac{\xi_{2}}{\xi_{L}}\right)^{C_{2}}=0.29
$$

where $\rho=4 / 3$ and $\xi_{2} / \xi_{L}=1.25$ was inserted. The result for $C_{2}=0.29$ is fairly insensitive under variation of $\xi_{2} / \xi_{L}$. For $1 \leq \xi_{2} / \xi_{L} \leq 1.5$, one obtains $0.27 \leq C_{2} \leq 0.31$. The value of $C_{2}=0.29$ is consistent with the experimental data, compare (3.18) and the corresponding theoretical curve in Fig. 11.

Imposing consistency between the CDP and the pQCD-improved parton model, we thus arrived at the prediction of a definite value of $C_{2}=0.29$ that coincides with the experimental findings in Fig. 11. 


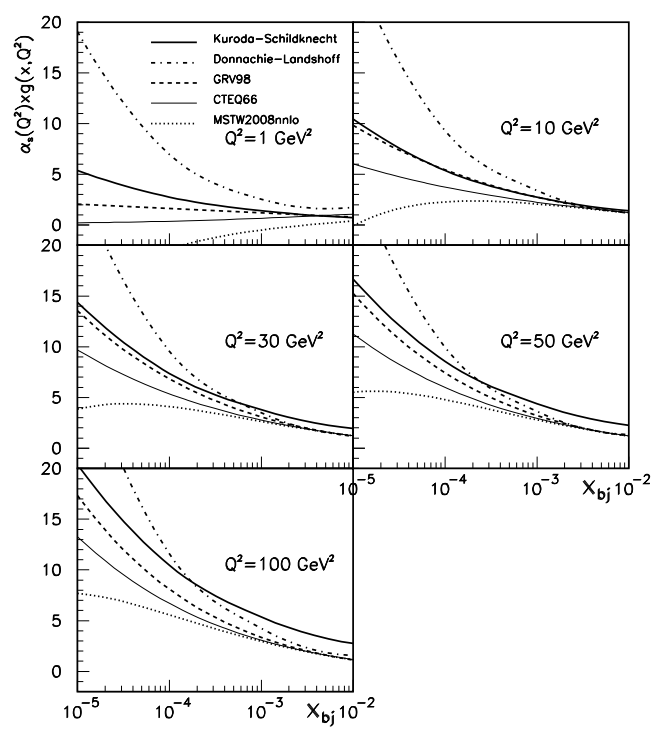

Figure 14: The gluon distribution function (4.8) compared with the results from the hard Pomeron part of a Regge fit to $F_{2}\left(x, Q^{2}\right)$ and from the fits GRV [41] CTEQ [42] and [43].

The underlying gluon distribution function can now be deduced from (4.1) by expressing the longitudinal structure function in terms of $F_{2}\left(x, Q^{2}\right)$ according to (3.17) and inserting the power law (3.18),

$$
\alpha_{s}\left(Q^{2}\right) G\left(x, Q^{2}\right)=\frac{3 \pi}{\sum_{q} Q_{q}^{2}(2 \rho+1)} \frac{f_{2}}{\xi_{L}^{C_{2}=0.29}}\left(\frac{W^{2}}{1 \mathrm{GeV}^{2}}\right)^{C_{2}=0.29} .
$$

With $\rho=4 / 3$ from the CDP, compare $(3.16)$, and $\sum_{q} Q_{q}^{2}=10 / 9$, the result 4.8 contains the single fitted parameter $f_{2}=0.063$ from (3.18). Inserting $W^{2}=Q^{2} / x$ on the right-hand side in (4.8), we obtain the gluon distribution as a function of $x$ and $Q^{2}$.

Using the next-to-leading order expression for $\alpha_{s}\left(Q^{2}\right)$, in Fig. 14 [4], we compare the gluon distribution 4.8 with various gluon distributions obtained in sophisticated global fits [41, 42, 43] to the experimental data. The consistency of our simple one-free-parameter extraction of the gluon distribution in Fig. 14 according to 4.8 may indicate that the gluon distribution is less sensitively dependent on the details of the ggpp structure than usually assumed, or elaborated upon and employed in the global fits to the experimental data.

\section{Specific ansatz for the dipole cross section and com- parison with experiment}

Any specific ansatz for the dipole cross section has to interpolate between the colortransparency region of $\eta\left(W^{2}, Q^{2}\right) \gg 1$, where $\sigma_{\gamma^{*} p}\left(\eta\left(W^{2}, Q^{2}\right)\right) \sim 1 / \eta\left(W^{2}, Q^{2}\right)$, and the saturation region of $\eta\left(W^{2}, Q^{2}\right) \ll 1$, where $\sigma_{\gamma^{*} p}\left(\eta\left(W^{2}, Q^{2}\right)\right) \sim \ln \left(1 / \eta\left(W^{2}, Q^{2}\right)\right)$, compare (3.13). For the explicit expressions for the ansatz for the dipole cross section, we refer to refs. [1] and [4] 6. We only note that the saturation scale, $\Lambda_{\text {sat }}^{2}\left(W^{2}\right) \sim\left(W^{2}\right)^{C_{2}}$ in

\footnotetext{
${ }^{6}$ For deeply virtual Compton scattering and vector meson production in the CDP compare ref. [44]
} 
the HERA energy range approximately varies between $2 G e V^{2} \leq \Lambda_{\text {sat }}^{2}\left(W^{2}\right) \leq 7 G e V^{2}$, and restrict ourselves to presenting a comparison with the experimental data.
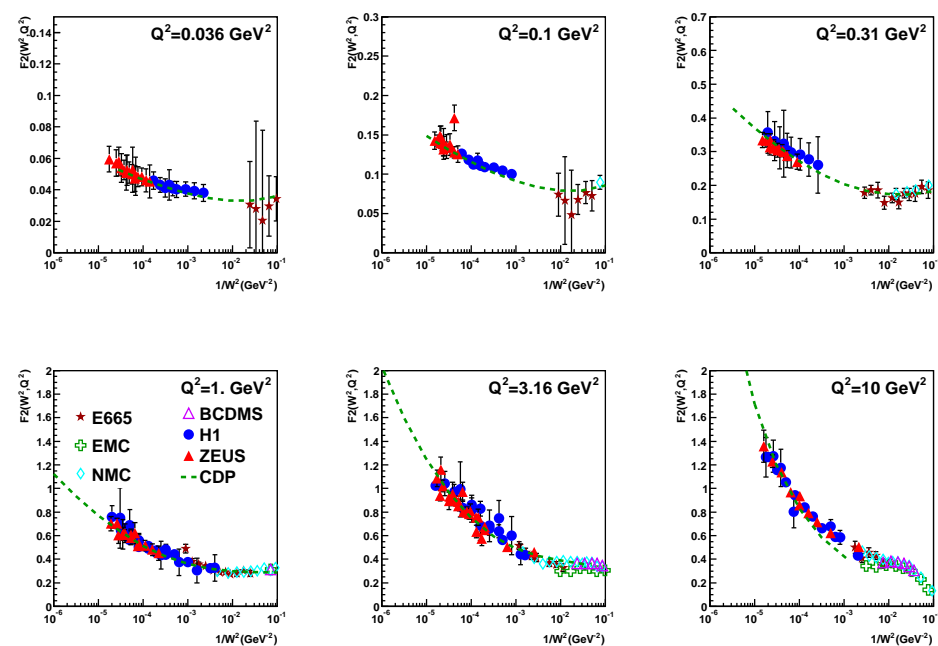

Figure 15: The predictions [4] from the CDP for the structure function $F_{2}\left(W^{2}, Q^{2}\right)$ compared with the experimental data for $0.036 \leq Q^{2} \leq 10 \mathrm{GeV}^{2}$.
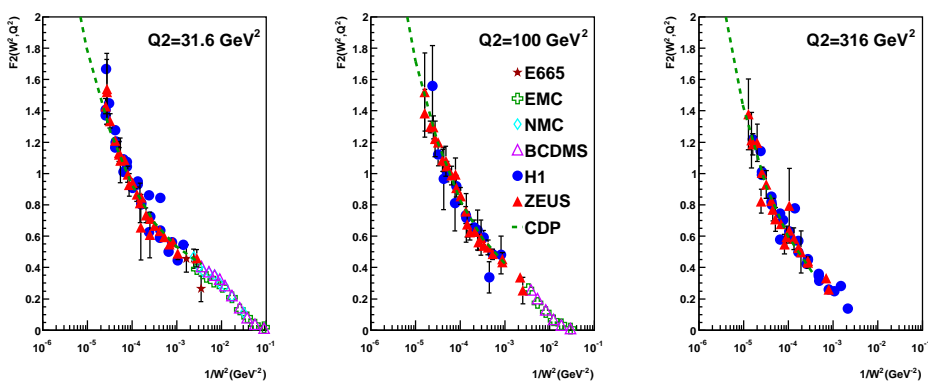

Figure 16: As in Fig. 15, but for $31.6 \mathrm{GeV}^{2} \leq Q^{2} \leq 316 \mathrm{GeV}^{2}$.

The theoretical results from the CDP in Figs. 15 and 16 show agreement with the experimental data 7 for $F_{2}\left(W^{2}, Q^{2}\right)$ over the full relevant region of $0.036 \mathrm{GeV}^{2} \leq Q^{2} \leq$ $316 \mathrm{GeV}^{2}$.

In Figs. 17 and 18, in addition to the theoretical results from the CDP, for comparison, we also show the results from the pQCD improved parton model based on the gluon distribution function (4.8) shown in Fig. 14.

Explicitly, by returning to (3.18) and inverting (4.8), we reinterprete (4.8) as a prediction of $F_{2}\left(W^{2}=Q^{2} / x\right)$ from the (previously determined) gluon distribution according to

$$
F_{2}\left(W^{2}=Q^{2} / x\right)=\frac{(2 \rho+1) \sum_{q} Q_{q}^{2}}{3 \pi} \xi_{L}^{C_{2}=0.29} \alpha_{s}\left(Q^{2}\right) G\left(x, Q^{2}\right),
$$

where $f_{2}=0.063$ and $\xi_{L}=0.40$. In Figs. 17 and 18, we see the expected consistency of the pQCD prediction (5.1) with the experimental data and the CDP in the relevant

\footnotetext{
${ }^{7}$ We thank Prabhdeep Kaur for providing the plots of the experimental data in Figs 15 to 18.
} 
range of $10 \mathrm{GeV}^{2} \leq Q^{2} \leq 100 \mathrm{GeV}^{2}$. For $Q^{2}<10 \mathrm{GeV}^{2}$, with increasing $W$, gradually saturation sets in implying a breakdown of the pQCD proportionality (5.1) of the proton structure function to the gluon distribution.
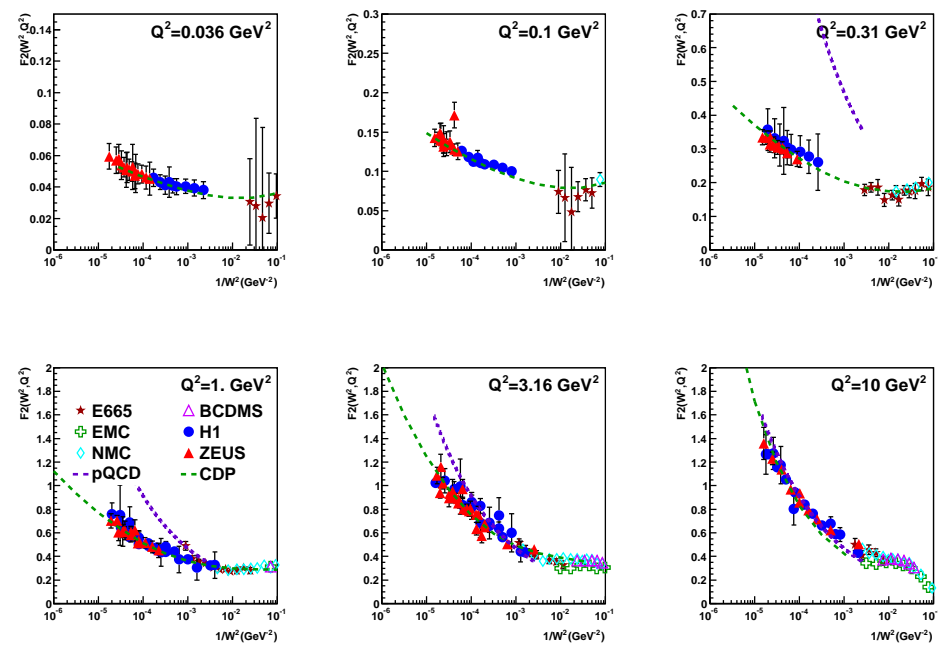

Figure 17: In addition to the prediction from the CDP, also the pQCD prediction (5.1) based on the gluon distribution (4.8) is compared with the experimental data for $F_{2}\left(W^{2}, Q^{2}\right)$.
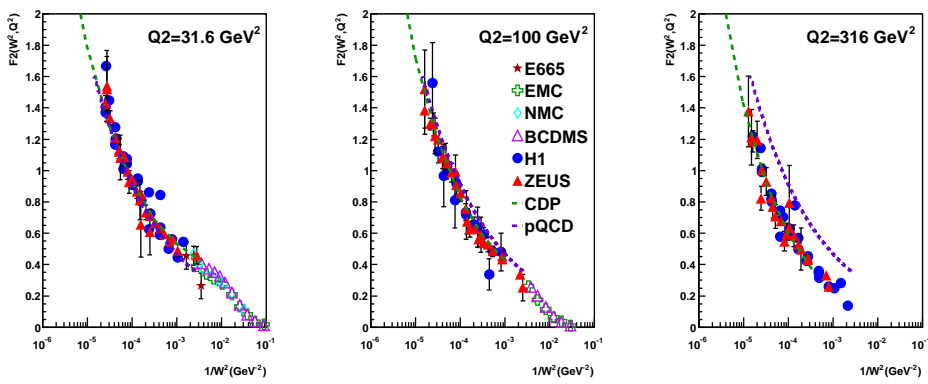

Figure 18: As in Fig. 17, but for $31.6 G e V^{2} \leq Q^{2} \leq 316 G e V^{2}$.

The pQCD prediction for $Q^{2}=316 G e V^{2}$ lies above the experimental data. This is due to the breakdown of the simple form for $F_{2}\left(W^{2}\right)$ in $(3.18)$ which is used to extract the gluon distribution. Employing the full CDP result would lead to an appropriate decrease of the gluon distribution with increasing $Q^{2}$ for $Q^{2}>100 \mathrm{GeV}^{2}$.

The proton structure function of the CDP, according to (3.13), in the region of $\eta\left(W^{2}, Q^{2}\right)<1$, starts to depend logarithmically on the saturation scale, $\Lambda_{\text {sat }}^{2}\left(W^{2}\right) \sim$ $\left(W^{2}\right)^{C_{2}}$, and with $\alpha_{s}\left(Q^{2}\right) G\left(x, Q^{2}\right) \sim\left(W^{2}\right)^{C_{2}}$ from (4.8) and (5.1), it depends logarithmically on the gluon distribution,

$F_{2}\left(W^{2}, Q^{2}\right) \sim Q^{2} \sigma_{L}^{(\infty)} \ln \frac{\Lambda_{s a t}^{2}\left(W^{2}=Q^{2} / x\right)}{Q^{2}+m_{0}^{2}} \sim Q^{2} \sigma_{L}^{(\infty)} \ln \frac{\alpha_{s}\left(Q^{2}\right) G\left(x, Q^{2}\right)}{\sigma_{L}^{(\infty)}\left(Q^{2}+m_{0}^{2}\right)},\left(Q^{2} \ll \Lambda_{s a t}^{2}\left(W^{2}\right)\right)$. 
The smooth transition from the color transparency region to the saturation region does not correspond to a change of the functional form of the $W$-dependent gluon distribution, $\alpha_{s}\left(Q^{2}\right) G\left(x, Q^{2}\right) \sim \Lambda_{\text {sat }}^{2}\left(W^{2}\right)$, but occurs via transition from the proportionality (5.1) to the logarithmic dependence (5.2) of $F_{2}\left(W^{2}, Q^{2}\right)$ on the gluon distribution function. It is the same gluon distribution that is relevant in the region of color transparency $\eta\left(W^{2}, Q^{2}\right) \gg 1$ and in the saturation region, $\eta\left(W^{2}, Q^{2}\right) \ll 1$, only the functional dependence of the proton structure function $F_{2}\left(x, Q^{2}\right)$ on the gluon distribution has changed. We disagree with the frequently expressed opinion (compare e.g. ref. [7] and the list of references given therein) that the mere existence of the saturation scale and of scaling like in Fig. 1 suggests and even requires a modification based on non-linear evolution of the gluon distribution determined in the pQCD domain of $\eta\left(W^{2}, Q^{2}\right)>1$, when passing to the saturation region of $\eta\left(W^{2}, Q^{2}\right)<1$.
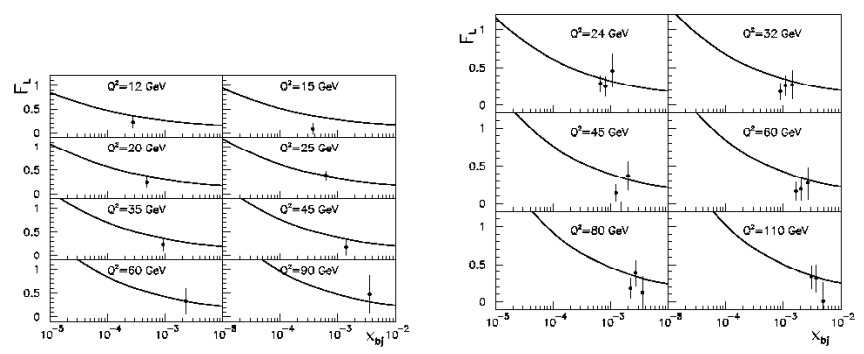

Figure 19: The longitudinal proton structure function $F_{L}\left(x, Q^{2}\right)$ from the H1 collaboration [45] and from the ZEUS collaboration [46] compared with the CDP prediction.

In Fig. 19, we show the experimental results [45, 46] for the longitudinal structure function, $F_{L}\left(x, Q^{2}\right)$, in comparison with the predictions from the CDP based on the specific ansatz for the dipole cross section that was used in Figs. 15 to 18.

\section{Conclusions}

The color-gauge-invariant interaction of a $q \bar{q}$ dipole state with the nucleon in terms of the forward scattering amplitude proceeds via two reaction channels. They correspond to the two diagrams shown in Fig. 9. For a given dipole size, if both reaction channels are open, the cancellation between them implies color transparency. In the limit in which the reaction channel corresponding to the second diagram in Fig. 9 is closed, the dipole cross section saturates to a cross section of standard hadronic size with at most a weak energy dependence.

In the photoabsorption cross section, the above limits of color transparency and saturation are realized, respectively, by the two regions in the $\left(Q^{2}, W^{2}\right)$ plane of Fig. 2 , corresponding to $\eta\left(W^{2}, Q^{2}\right) \gg 1$ and $\eta\left(W^{2}, Q^{2}\right) \ll 1$. The $\left(Q^{2}, W^{2}\right)$ plane (under the restriction of $\left.Q^{2} / W^{2} \lesssim 0.1\right)$ is accordingly simple. There are only two distinct regions in the $\left(Q^{2}, W^{2}\right)$ plane, separated by the line $\eta\left(W^{2}, Q^{2}\right)=1$.

The main features of the experimental data on DIS at low $\mathrm{x}$ have thus been recognized to follow from the color-gauge-invariant $q \bar{q}$ dipole-interaction with the gluon field of the proton without adopting a specific ansatz for the dipole cross section. Any specific ansatz 
has to interpolate between the model-independent restrictions on the photoabsorption cross section that hold for $\eta\left(W^{2}, Q^{2}\right) \gg 1$ and for $\eta\left(W^{2}, Q^{2}\right) \ll 1$, respectively.

\section{Acknowledgement}

The author thanks Kuroda-san for a fruitful collaboration on the color dipole picture. Thanks to Prabhdeep Kaur for providing plots of experimental data. The author thanks Allen Caldwell and Reinhart Kögerler for useful discussions.

Last not least the author thanks Professor Antonino Zichichi for the invitation to the 50th Anniversary Celebrations of his famous School of Subnuclear Physics with its lively scientific atmosphere in the magnificent environment of the "Ettore Majorana" Foundation and Centre for Scientific Culture at Erice, Sicily.

\section{References}

[1] D. Schildknecht, Contribution to Diffraction 2000, Cetraro, Italy, September 2-7, 2000, Nucl. Phys. B, Proc. Supplement 99 (2001) 121;

D. Schildknecht, B. Surrow, M. Tentyukov, Phys. Lett. B499 (2001) 116;

G. Cvetic, D. Schildknecht, B. Surrow, M. Tentyukov, EPJC 20 (2001) 77.

[2] D. Schildknecht, Contribution to DIS 2001, The 9th International Workshop on Deep Inelastic Scattering, Bologna, Italy, 2001, G. Brassi et al. (Eds.), World Scientific, Singapore, 2002, p. 798;

D. Schildknecht, B. Surrow and M. Tentyukov, Mod. Phys. Lett. A16 (2001) 1829.

[3] M. Kuroda and D. Schildknecht, Phys. Lett. B670 (2008) 129.

[4] M. Kuroda and D. Schildknecht, Phys. Rev. D85 (2012) 094001.

[5] M. Kuroda and D. Schildknecht, Phys. Lett. B618 (2005) 84.

[6] D. Schildknecht, arXiv:1104.0850 [hep-ph]]

[7] J. Kuokkanen, K. Rummukainen and H. Weigert, [arXiv:1108.1867 [hep-ph]].

[8] J.J. Sakurai, Ann. of Physics 11 (1960) 1; Currents and Mesons, The University of Chicago Press, Chicago 1969.

[9] M. Gell-Mann and F. Zachariasen, Phys. Rev. 124 (1961) 953.

[10] N.M. Kroll, T.D. Lee and B. Zumino, Phys. Rev. 157 (1967) 1376.

[11] D. Schildknecht, Springer Tracts in Modern Physics, Vol. 63 (1972) p. 57.

[12] D. Schildknecht, in Proceedings of Photon 2005, International Conference on the Structure and Interaction of the Photon, Warsaw 2005, Acta Phys. Polon. B37 (2006) 595 hep-ph/0511090. 
[13] J.J. Sakurai, Dynamical Models Based on Vector Meson Dominance and Universality Properties of the Fundamental Interactions, in Properties of the Fundamental Interactions, 1971 International School of Subnuclear Physics, Erice, Sicily (ed. A. Zichichi) Editrice Compositori, Bologna (1973), p. 243.

[14] J.J. Sakurai, Hadronlike Behaviour of the Photon, in Laws of Hadronic Matter, 1973 International School of Subnuclear Physics, Erice, Sicily (ed. A. Zichichi) Academic Press New York and London (1975).

[15] L. Stodolsky, Phys. Rev. Lett. 18 (1967) 135;

S.J. Brodsky and J. Pumplin, Phys. Rev. 182 (1969) 1794;

M. Nauenberg, Phys. Rev. Lett. 22 (1969) 556;

K. Gottfried and D.R. Yennie, Phys. Rev. 182 (1969) 1595.

[16] SLAC-MIT Collaboration, G. Miller et al., Phys. Rev. D5 (1972) 528.

[17] J.D. Bjorken, Phys. Rev. 179 (1969) 1547.

[18] R.P. Feynman, Photon Hadron Interactions, Benjamin, New York (1972).

[19] J.J. Sakurai and D. Schildknecht, Phys. Lett. 40B (1972) 121; Phys. Lett. 41B (1972) 489; Phys. Lett. 42B (1972) 216;

B. Gorczyca, D. Schildknecht, Phys. Lett. 47B, 71 (1973).

[20] C. Bernardini, in Proc. 5th Internat. Symp. on Electron and Photon Interactions at High Energies, Cornell University (1971)

[21] H. Fraas, B.J. Read and D. Schildknecht, Nucl. Phys. B86 (1975) 346;

R. Devenish and D. Schildknecht, Phys. Rev. D19 (1976) 93.

[22] D. Schildknecht, Is Vector Dominance Still Alive?, in Lepton and Hadron Structure, 1974 Int. School of Subnuclear Physics, Erice, Sicily, 14-31. July 1974 (ed. A. Zichichi), Academic Press New York and London, p. 448.

[23] EMC-NMC Collaboration, compare: M. Arneodo, Phys. Rep.240 (1994) 301.

[24] D. Schildknecht, Nucl. Phys. B66 (1973) 398;

C. Bilchak and D. Schildknecht, Phys. Lett. B214 (1988) 441; Phys. Lett. B233 (1989) 461.

[25] H1 Collaboration, T. Ahmed et al., Nucl. Phys. B429 (1994) 477; ZEUS Collaboration, M. Derrick et al., Phys. Lett. B315 (1993) 481; R. Wichmann, on behalf of the ZEUS and H1 Collaborations, Nucl. Phys. B (Proc. Suppl.) 82 (2000) 268.

[26] T. Nieh, Phys. Rev. D1 (1970) 3161;

B.L. Joffe, Phys. Lett. 30B (1969) 123;

V.N. Gribov, Sov. Phys.-JETP 30 (1970) 709. 
[27] F.E. Low, Phys. Rev. D12 (1075) 163;

S. Nussinov, Phys. Rev. Lett. 34 (1975) 1286; Phys. Rev. D14 (1976) 246;

J. Gunion, D. Soper, Phys. Rev. D15 (1977) 2617.

[28] N.N. Nikolaev, B.G. Zakharov, Z. Phys. C49, 607 (1991).

[29] G. Cvetic, D. Schildknecht, A. Shoshi, Eur. Phys. J C13 (2000) 301.

[30] J.R. Forshaw, G. Kerley, G. Shaw, Phys. Rev. D60 (1999) 074012.

[31] C. Ewerz and O. Nachtmann, Annals of Phys. 322 (2007) 1635; 322 (2007) 1670;

C. Ewerz, A.v. Manteuffel, O. Nachtmann, arXiv:1101.0288 [hep-ph]].

[32] M. Kuroda and D. Schildknecht, Phys. Rev. D66(2002) 094005; Phys. Rev. D67 (2003) 094008.

[33] D. Schildknecht and F. Steiner, Phys. Lett. B56 (1975) 36;

J.J. Sakurai, Phys. Lett. B46 (1973) 207, and New Duality in Electromagnetic Interactions, 1973 International School of Subnuclear Physics (ed. A. Zichichi) loc. cit. p. 291

[34] C. Ewerz, A.v. Manteuffel, O. Nachtmann, A. Schöning, arXiv: 1201.6296 [hep-ph]

[35] A. Caldwell, arXiv:0802.0769

[36] A.D. Martin, R.G. Roberts and W.J. Stirling, Phys. Rev. D37 (1988) 1161;

A.M. Cooper-Sarkar et al., Z. Phys. C39 (1988) 281.

[37] L.N. Lipatov, Sov. J. Nucl. Phys. 20 (1975) 95,

V.N. Gribov and L.N. Lipatov, Sov. J. Nucl. Phys. 15 (1972) 438,

G. Altarelli and G. Parisi, Nucl. Phys. B126 (1977) 298;

Yu. L. Dokshitzer, Sov. Phys. JETP 46 (1977) 641.

[38] K. Prytz, Phys. Lett. B311 (1993) 286.

[39] K. Adel, F. Barreiro, F.J. Yndurain, Nucl. Phys. B495 (1997) 221;

F.J. Yndurain, The Theory of Quark and Gluon Interactions (Springer 1999), p.157.

[40] A. Donnachie and P.V. Landshoff, Phys. Lett. B533 (2002) 277, hep-ph/0111427; Acta Physica Polonica B34 (2003) 2989, hep-ph/0305171].

[41] M. Glück, E. Reya, A. Vogt, Z. Phys. C67 (1994) 433; Eur. Phys. J. C5 (1998) 461

[42] CTEQ Collaboration: J. Pumplin et al., JHEP 0207 (2002) 012.

[43] A.D. Martin et al., Eur. Phys. J. C18 (2000) 117.

[44] M. Kuroda and D. Schildknecht, Eur. Phys. J. C37 (2004) 205; Erratum, ibid. C44 (2005) 613; Phys. Lett. B618 (2005) 84.

[45] H1 Collaboration, F.D. Aaron et al., Phys. Lett. B665 (2008) 139.

[46] ZEUS Collaboration, S. Chekanov et al., Phys. Lett. B682 (2009) 8. 